\title{
Cell engineering by the internalization of bioinstructive micelles for enhanced bone regeneration
}



\begin{abstract}
Aim: To direct precursor cells toward the osteoblastic lineage, by using an intracellular nanocarrier releasing dexamethasone. Materials \& methods: Biodegradable gelatinbased micelles entrapped dexamethasone (dex-micelles). Internalization efficiency and biocompatibility of dex-micelles and their potency for in vitro osteogenic differentiation and in vivo bone regeneration were assessed. Results: Dex-micelles were internalized by rat bone marrow mesenchymal stem cells and demonstrated a $\mathrm{pH}$-responsive release profile and an enhancement of 2D and 3D in vitro osteogenic differentiation. In vivo implantation of gelatin scaffolds seeded with rat bone marrow mesenchymal stem cells precultured for $24 \mathrm{~h}$ with dex-micelles promoted a significant enhancement of de novo bone formation in a rat ulna defect, in a dose-dependent manner. Conclusion: The proposed intracellular delivery system is a powerful tool to promote bone regeneration.
\end{abstract}

Keywords: bone regeneration $\bullet$ intracellular delivery $\bullet$ mesenchymal stem cell $\bullet$ micelle

- nanoparticle • tissue engineering

Bone marrow-derived mesenchymal stem cells (BMSCs) have been widely investigated and their osteogenic differentiation potential and bone regeneration capability in both animal [1,2] and human models [3-5] has been consistently demonstrated. The osteogenic differentiation of BMSCs can be induced by soluble factors such as dexamethasone (dex), typically present in the osteogenic media cocktail used for in vitro cultures [6].

Dex is a non-water-soluble synthetic glucocorticosteroid that can induce bone formation through the triggering of a distinct set of pathways, including chemokine and calcium signaling $[7,8]$. The stimulatory role of dex has been reported at early stages of osteogenic differentiation [7,9-10] but also at late stages of differentiation, directing cells toward terminal maturation through the formation of mineralization nodules [11,12]. Dex acts through the modulation of nuclear steroid receptors [7], initiating transduction mechanisms which ultimately will lead to bone formation. It is described in the litera- ture that dex regulates Runx2 via FHL2/ $\beta$ catenin-mediated transcriptional activation [13], via activation of TAZ [14] and via activation of MKP-1 [10]. Dex has also been shown to invariably increase the proportion of cells expressing alkaline phosphatase (ALP), an early osteogenic cell marker [15]. In summary, dex regulation depends on the degree of cellular differentiation, the donor species, dosage, dose duration and dosing regimen $[8,16]$.

Cell engineering and tissue engineering have benefited from the development of novel strategies that can stimulate and control cell functions both in vitro and in vivo [17,18]. One possible approach could be through the use of drug delivery systems promoting the enhancement of cell proliferation and differentiation and the production of extracellular matrix (ECM) [19]. The design of novel nanomaterials, such as polymeric micelles, to act as carriers for controlled release is one of the various strategies applied to overcome this challenge [20]. Polymeric micelles are nano-
Vítor E Santo ${ }^{1,2,3}$, Juthamas Ratanavaraporn', Keisuke Sato', Manuela E Gomes ${ }^{2,3}$, João F Mano ${ }^{2,3}$, Rui L Reis*,2,3 \& Yasuhiko Tabata'

'Department of Biomaterials, Field of Tissue Engineering, Institute for Frontier Medical Sciences, Kyoto University, Sakyo Ku, Kyoto 6068507, Japan 23B's Research Group - Biomaterials, Biodegradables \& Biomimetics, University of Minho, Headquarters of the European Institute of Excellence on Tissue

Engineering \& Regenerative Medicine, AvePark, 4806-909 Taipas, Guimarães, Portugal

${ }^{3}$ CCVS/3B's - PT Government Associate Laboratory, Braga/Guimarães, Portugal *Author for correspondence:

Tel.: +351 253510900

Fax: +351253510909

rgreis@dep.uminho.pt 
sized supramolecular constructs formed from the self assembly of amphiphilic copolymers in aqueous environments with the ability to carry and to deliver hydrophobic drugs [21]. These micelles can be tailored for intracellular drug release, according with the desired experimental design and location of the specific drug receptors [22].

The internalization of the nanoparticles by the cells allows the modulation of the activity of intracellular receptors such as those described for corticosteroids [23]. Previous studies [12,24] reported the delivery of dex from polymeric carriers, resulting in an enhancement of osteogenesis. However, the majority focused on an extracellular delivery of the drug with few exceptions, such as the work of Oliveira et al. [16,25], which has showed the potency of intracellular release of dex in order to further enhance osteogenic differentiation of BMSCs.

In this work we propose the application of a gelatin-based micelle system for entrapment and intracellular release of dex. The gelatin polymer chain was grafted with lactic acid oligomers in order to create a hybrid amphiphilic, able to solubilize dex in an aqueous environment (dex-micelles). We hypothesize that the internalization of the drug-loaded micelles might enhance the in vivo osteogenic potential of the transplanted rat BMSCs (rBMSCs), thus avoiding the withdrawal of dex post in vitro preculture and reducing the preculture period.

The specific aims of this study were threefold: to assess the potential of gelatin-based micelles to be used as a carrier for the intracellular delivery of dex (Supplementary Figure 1A; please see online at http://www.futuremedicine.com/doi/full/10.2217/ NNM.15.11); to evaluate the effect of the dex-micelles internalization on the in vitro osteogenic differentiation of a rat preosteoblastic cell line (MC3T3-E1) and rBMSCs (Supplementary Figure 1B); to assess the ability of gelatin scaffold constructs seeded with rBMSCs precultured with the dex-micelles to heal critical-sized bone defects, using a rat ulna defect model (Supplementary Figure 1C). Healing rates were evaluated my measuring new bone formation within the defect using radiological, micro-CT, histological and immunohistochemistry techniques.

\section{Materials \& methods \\ Materials}

A gelatin sample prepared by an acidic treatment of porcine skin collagen (isoelectric point $[\mathrm{IEP}]=9.0$ ) was kindly supplied by Nitta Gelatin, Inc. (Osaka, Japan). Disuccinimidyl carbonate (DSC) and 4-dimethylaminopyridine (DMAP) were obtained from Nacalai Tesque, Inc., Kyoto, Japan. Glutaraldehyde (GA), glycine, DMSO and other chemicals were obtained from
Wako Pure Chemical Industries Ltd, Osaka, Japan and used without further purification.

\section{Synthesis of L-lactic acid oligomer-grafted gelatin}

L-lactic acid oligomer $\left(\mathrm{LA}_{\mathrm{o}}\right)$ with an average molecular weight of $1000 \mathrm{D}$ was synthesized from L-lactide monomer by the ring-opening polymerization as reported in a previous study [26]. Briefly, L-lactic acid $\left(3 \times 10^{-5} \mathrm{~mol}\right)$ was dissolved in $15 \mathrm{ml}$ DMSO while DSC $\left(9 \times 10^{-5} \mathrm{~mol}\right)$ and DMAP $\left(9 \times 10^{-5} \mathrm{~mol}\right)$ were dissolved in $2.5 \mathrm{ml}$ of DMSO. The solution was mixed to allow the reaction for $3 \mathrm{~h}$ under stirring at room temperature to activate the hydroxyl groups of LA. The solution of activated oligomer was slowly added to gelatin $($ IEP $=5)$ solution in DMSO $(33 \mathrm{mg} / \mathrm{ml})$ and the mixture was stirred overnight at room temperature to promote the grafting of $\mathrm{LA}_{\mathrm{o}}$ to gelatin. The product solution was dialyzed against double distilled water with a dialysis tube (molecular weight cut off $=12,000-14,000)$ for $72 \mathrm{~h}$ at room temperature, followed by freeze drying to obtain the LA -grafted gelatin. The ratio of LA grafted to the amino groups of gelatin determined by the conventional fluorescamine assay was $3.1 \pm 0.8 \mathrm{~mol} / \mathrm{mol}$ gelatin.

\section{Preparation of dex-micelles}

LA -grafted gelatin solution $(1 \mathrm{mg} / \mathrm{ml})$ in DMSO and a dex solution $(1.0 \mathrm{mg} / \mathrm{ml})$ in DMSO were prepared. The dex solution $(15 \mathrm{ml})$ was added to the $\mathrm{LA}_{\mathrm{o}}-$ grafted gelatin solution $(30 \mathrm{ml})$, followed by $3 \mathrm{~h}$ stirring at room temperature. The reaction mixture was dialyzed with a dialysis tube (molecular weight cut off $=1000 \mathrm{D}$ ) against double distilled water for $72 \mathrm{~h}$ at room temperature. The dialysate obtained was centrifuged $\left(8000 \mathrm{rpm}, 10 \mathrm{~min}, 4^{\circ} \mathrm{C}\right)$ to remove water-insoluble dex, and freeze dried to obtain the dex water solubilized by LA -grafted gelatin micelles (dex-micelles). In order to measure the amount of dex incorporated into the dex-micelles, the freeze-dried dex-micelles were dissolved in $100 \mathrm{vol} \%$ ethanol. The solution was sonicated for several cycles and centrifuged at $5000 \mathrm{~g}$ for $10 \mathrm{~min}$. The supernatant was quantified in a HPLC (LC-20AT, Shimadzu, Japan) run in a solution composed by sample/acetonitrile/acetate buffer/acetic acid (50/25/24/1 vol\%). An analytical column (TSKgel ODS-100V 5um, TOSOH, Japan) was used and the retention time for dex was approximately $4 \mathrm{~min}$. Dex concentration was determined from the standard curve prepared with the ethanol containing various amounts of dex and dispersed in the same running solution of sample/acetonitrile/acetate buffer/acetic acid (50/25/24/1 vol\%). The drug loading achieved was of $15 \mathrm{ng}$ dex per $\mu \mathrm{g}$ of dex-micelles. 


\section{Morphological characterization of the}

dex-micelles

The morphological analysis of the developed micelles was performed by atomic force microscopy (AFM). A drop of dex-micelles suspension of about $0.001 \%(\mathrm{w} / \mathrm{v})$ was placed on a silicon wafer square $(10 \times 10 \mathrm{~mm})$ and AFM measurements were performed using a MultiMode STM Microscope controlled by the NanoScope III from Digital Instruments system operating in the tapping mode at a frequency of $1 \mathrm{~Hz}$.

\section{Size \& surface charge measurements of dex-micelles}

The size and zeta potential of the dex-micelles were measured by photon correlation spectroscopy and laser Doppler anemometry, respectively, using a Malvern Zetasizer Nano ZS (Malvern Instruments, Malvern, UK). The size analysis was performed at $25^{\circ} \mathrm{C}$ with a detection angle of $90^{\circ}$ and the zeta potential was carried under an electric field strength of $100 \mathrm{~V} / \mathrm{cm}$. The measurements were performed with the dex-micelles dispersed in phosphate-buffered saline (PBS; $\mathrm{pH} 7.4$ ) and citrate buffer ( $\mathrm{pH} 4.0$ ). Each sample was diluted to the appropriate concentration with filtrated distilled water $(0.22 \mu \mathrm{m})$.

\section{In vitro dex release studies}

The release of dex was determined by incubating dex-micelles $(2 \mu \mathrm{g}$ entrapped dex $)$ in $1 \mathrm{ml}$ of $\mathrm{pH} 7.4$ phosphate buffer or $1 \mathrm{ml}$ of $\mathrm{pH} 4.0$ citrate buffer, with horizontal shaking, at $37^{\circ} \mathrm{C}$ under sink conditions $(100 \mu \mathrm{g} / \mathrm{ml}$ saturation solubility). At appropriate time intervals, the release medium was removed, centrifuged for $10 \mathrm{~min}$ at $8000 \mathrm{rpm}$ and the supernatant was collected, freeze dried and dissolved in ethanol. Following a new centrifugation step $(8000 \mathrm{rpm}$, $10 \mathrm{~min}, 4^{\circ} \mathrm{C}$ ), the dex amount in the supernatant was determined by HPLC analysis following the procedure previously described.

Labeling of the dex-micelles with rhodamine $B$ A $10 \mathrm{mg} / \mathrm{ml}$ Rhodamine $\mathrm{B}(\mathrm{RhD})$ solution was prepared in anhydrous DMSO, in dark conditions. Firstly, a $1 \mathrm{mg} / \mathrm{ml}$ dex-micelles solution was prepared in a carbonate-bicarbonate coupling buffer of $\mathrm{pH}$ 9.2. Then $5 \mu \mathrm{l}$ of the RhD/DMSO solution was added per each $\mathrm{ml}$ of micelles buffered solution under agitation, at $4^{\circ} \mathrm{C}$ for $8 \mathrm{~h}$. The Rhd-labeled micelles solution was dialyzed against double distilled water in order to remove the unlinked $\mathrm{RhD}$ for $48 \mathrm{~h}$ and the labeled micelles were frozen at $-80^{\circ} \mathrm{C}$ and freeze dried.

\section{Isolation \& culture of rBMSCs}

The rBMSCs were isolated from the bone shaft of femurs of 3-week-old Fischer 344 rats according to the procedure reported previously [27]. Briefly, after cutting off both ends of rat femurs, the bone marrow was flushed out with $1 \mathrm{ml}$ of alpha-modified eagle minimal essential medium ( $\alpha M E M)$. Then, the bone marrow cells mixture was cultured in aMEM supplemented with $15 \mathrm{vol} \%$ fetal bovine serum, and $100 \mathrm{U} / \mathrm{ml}$ penicillin and $100 \mu \mathrm{g} / \mathrm{ml}$ streptomycin at $37^{\circ} \mathrm{C}$ in a $5 \% \mathrm{CO}_{2}$ incubator. The medium was refreshed on the 4th day after isolation to remove nonadherent cells and continuously refreshed every 3 days thereafter. The cells of the second and third passages at the subconfluent condition were used for the following experiments.

\section{Evaluation of micelles internalization by MC3T3-E1 cells \& rBMSCs}

Mouse MC3T3-E1 osteoblast-like cells and rBMSCs were cultured with $\alpha$ MEM medium supplemented with 10 vol\% fetal bovine serum and $100 \mathrm{U} / \mathrm{ml}$ penicillin/100 $\mu \mathrm{g} / \mathrm{ml}$ streptomycin (complete basal medium) at $37^{\circ} \mathrm{C}$ in a $5 \% \mathrm{CO}_{2}$ incubator until reaching confluence. Three different concentrations of RhD-labeled micelles were added to the culture medium: $6.5,32.5$ and $65 \mu \mathrm{g}$ per million of cells, designated as low, medium and high doses of dex-micelles, respectively. The cells were incubated for 3, 8 and $24 \mathrm{~h}$ with the micelles. At each time point, the flasks were washed with PBS and the internalization efficiency was assessed by fluorescence microscopy and flow cytometry. The mechanism of internalization was assessed by incubating rBMSCs with $1 \mu \mathrm{m}$ colchicine for $24 \mathrm{~h}$ in the presence of high dose of dex-micelles, followed by further analysis through flow cytometry. For the flow cytometry analysis, cells were trypsinized and incubated for $5 \mathrm{~min}$ with propidium iodide, to distinguish dead from viable cells. The cells were then analyzed on FACSCanto II flow cytometer (BD Biosciences, Inc., MA, USA). Analysis was performed by BD FACSDiva software (BD Biosciences, Inc.). Each analysis was performed for three samples. The fluorescence images were taken with an Apotome, Imager Z1 fluorescence microscope (Carl Zeiss, Jena, Germany).

\section{In vitro MC3T3-E1 cell viability \& proliferation}

MC3T3-E1 cells were cultured in complete basal medium at $37^{\circ} \mathrm{C}$ in a $5 \% \mathrm{CO}_{2-} 95 \%$ air atmospheric condition until reaching confluence. A suspension of 12.500 cells in $500 \mu \mathrm{l}$ of culture medium was seeded onto each well in 24-well culture plates (Corning, Inc., MA, USA) and cultured for 1, 5, 7 and 14 days with either complete osteogenic medium or osteogenic medium without dex. The osteogenic medium was composed by complete basal medium supplemented with $50 \mu \mathrm{g} / \mathrm{ml}$ ascorbic acid, $10 \mathrm{mM} \beta$-glycerophosphate and $10^{-8} \mathrm{M}$ dex. Four different group studies were 
assessed for each culture medium: low dose of empty micelles; high dose of empty micelles; low dose of dex-micelles and high dose of dex-micelles.

Cytotoxicity of unloaded and dex-micelles was assessed using the Cell Count Reagent SF (Nacalai Tesque, Inc., Kyoto, Japan) as a colorimetric indicator for living cells. At each time point, $100 \mu \mathrm{l}$ of the reagent containing WST-8 $(5 \mathrm{mM})$ was added to each well containing the cells and incubated for $3 \mathrm{~h}$. Absorbance of each well was measured at $450 \mathrm{~nm}$ using a microplate reader. The assay was performed three-times independently for each experimental sample.

The number of cells was determined by the fluorometric quantification of cellular DNA. The cell lysates in $30 \mathrm{mM}$ sodium citrate-buffered saline solution (SSC, $\mathrm{pH} 7.4$ ) containing $0.2 \mathrm{mg} / \mathrm{ml}$ sodium dodecylsulfate at $37^{\circ} \mathrm{C}$ were mixed for $12 \mathrm{~h}$. The cell lysate $(100 \mu \mathrm{l})$ was mixed with the SSC buffer $(400 \mu \mathrm{l})$ in a glass tube. After mixing with a dye solution $(500 \mu \mathrm{l}$; $30 \mathrm{mM}$ SSC, $1 \mu \mathrm{g} / \mathrm{ml}$ Hoechst 33258 dye), the fluorescent intensity of mixed solution was measured in a fluorescence spectrometer (F-2000, HITACHI, Tokyo, Japan) at the excitation and emission wavelengths of 355 and $460 \mathrm{~nm}$, respectively. The calibration curve between the DNA and cell number was prepared by using cell suspensions of known concentration. The DNA assay was performed three-times independently for each experimental sample.

\section{In vitro 2D osteogenic differentiation}

MC3T3-E1 cells were seeded on 24 well culture plates by adding 12.500 cells in $500 \mu \mathrm{l}$ of culture medium per well. Cells were either cultured in complete osteogenic medium or osteogenic medium without dex up to 14 days. Four different group studies resulted from adding micelles at the beginning of the culture: low dose of empty micelles; high dose of empty micelles; low dose of dex-micelles and high dose of dex-micelles.

The activity of ALP of cells cultured in the hydrogels for 5,7 and 14 days was assayed by the conventional p-nitrophenyl phosphate method. The ALP activity was normalized by the number of cells, determined by the fluorometric quantification of cellular DNA. The experiment was independently performed for three samples per experimental group. For detecting possible ECM mineralization, samples cultured for 14 days were stained with alizarin red staining. For the mineralization assessment, the replacement of emptyand dex-micelles was also performed each 3 days, when the osteogenic medium was replaced.

\section{Preparation of gelatin hydrogels}

Gelatin hydrogels chemically crosslinked with glutaraldehyde were prepared according to the method pre- viously reported [28]. Briefly, $100 \mu \mathrm{l}$ aqueous solution of gelatin with IEP $=9.0(50 \mathrm{mg} / \mathrm{ml})$ was mixed with glutaraldehyde solution at a concentration of $0.16 \mathrm{vol} \%$ and cast into a polypropylene dish $(10 \times 10 \mathrm{~mm})$. The gelatin crosslinking occurred for the following $12 \mathrm{~h}$ at $4^{\circ} \mathrm{C}$. The crosslinked gelatin hydrogels were treated with $0.1 \mathrm{M}$ aqueous glycine solution at room temperature for $2 \mathrm{~h}$ to block the residual aldehyde groups of glutaraldehyde. Following a three-time washing with double-distilled water, the hydrogels were freeze dried and cut into the appropriate dimensions $\left(1 \times 2 \times 6 \mathrm{~mm}^{3}\right)$. Before every experiment, the hydrogels were sterilized by ethylene oxide.

\section{In vitro 3D osteogenic differentiation}

MC3T3-E1 cells were cultured in complete basal medium. The medium was refreshed every 3 days and cells were trypsinized when reaching $80 \%$ confluence in the culture flasks. At this point, cells were incubated for $24 \mathrm{~h}$ with three different dosages of dex-micelles (low, medium and high dose), with free-form dex (in the concentration of typical osteogenic medium $10^{-8} \mathrm{M}$ ) and with PBS only, as negative control.

After $24 \mathrm{~h}$ of in vitro culture, the flasks were washed twice with PBS to remove noninternalized micelles and cells were then trypsinized and seeded onto gelatin hydrogels. A suspension of 1 million cells/50 $\mu \mathrm{l}$ was seeded into each hydrogel for $6 \mathrm{~h}$ by the agitation seeding technique reported previously. Further, $1 \mathrm{ml}$ of the medium was added to each construct and static culture in osteogenic medium without dex was carried on.

The activity of ALP for cells cultured in the hydrogels for 5, 7 and 14 days was quantified as previously described. The ALP activity was normalized by the number of cells, determined by the fluorometric quantification of cellular DNA. The experiment was independently performed for three samples per experimental group.

\section{In vivo bone regeneration in a rat ulna critical-sized defect}

A bone defect model of rat ulna was used to evaluate the bone regeneration induced by implantation of hydrogels seeded with rBMSCs precultured with dex-micelles or free-form dex and rBMSCs not precultured. Surgery was made for 12-week-old male Fisher 344 rats $(\mathrm{n}=24)$ under standard sterile conditions according to the procedure previously reported [29]. All the animal experiments were performed following the Institutional Guidance of Kyoto University on Animal Experimentation and upon approval of the animal experiment committee of the Institute for frontier Medical Science, Kyoto University. 
The constructs implanted consisted of gelatin hydrogels seeded with 1 million rBMSCs precultured for $24 \mathrm{~h}$ with low, medium and high doses of dex-micelles. One additional group study consisted of hydrogels seeded with 2 million of rBMSCs precultured with high dose of dex-loaded micelles. Hydrogels with precultured cells with free-form dex or PBS alone were used as controls. Each rat received the implantation of two constructs randomly placed in both left and right of forearm's ulna.

Briefly, the rats were anesthetized with an intraperitoneal injection of pentobarbital sodium solution (35-40 $\mathrm{mg} / \mathrm{kg}$ bodyweight). After shaving the hair and disinfection with $70 \mathrm{vol} \%$ ethanol, a longitudinal incision was made along the forearm skin of rats. The periosteum was incised circumferentially to approach to the ulna bone. A critical defect of $6 \mathrm{~mm}$ in length was then created at the middle position of ulna bone using a side-cutting diamond disk and a high-speed micromotor under an abundant irrigation with sterile saline solution. The hydrogels were implanted into the defects while the periosteum and overlying muscle were repositioned with an absorbable polydioxanone suture (Ethicon 7-0, NJ). Then, the wound was closed with a nonabsorbable polypropylene suture (Ethicon 4-0, NJ).

\section{Evaluation of bone tissue regenerated}

The bone tissues regenerated at the defect and subcutaneous site, 2 and 4 weeks later, were radiologically examined by soft x-ray machine (Hitex-100, Hitachi Ltd, Tokyo, Japan) at $56 \mathrm{kV}$ and $2.5 \mathrm{~mA}$ for $20 \mathrm{~s}$. Then, the samples were kept at $4 \mathrm{wt} \%$ paraformaldehyde in PBS at $4^{\circ} \mathrm{C}$, until further analysis. 3D images of bone regenerated in the ulna defects were visualized with the CT scans (Skyscan 1072, Belgium). Samples were scanned at a resolution of $19.12 \mu \mathrm{m}$, energy of $100 \mathrm{kV}$, current of $98 \mu \mathrm{A}$ and exposure time of $2464 \mathrm{~ms}$. The $\mathrm{x}$-ray scans were acquired in high resolution mode. The 2D images were reconstructed with CT Analyzer (Skyscan, Belgium) to produce the $3 \mathrm{D}$ images in the region of the bone defect. Analysis of new bone volume in the defect zone was carried out within a circular region of interest with at least 140 tomographic layers. Then, the fixed samples were decalcified and included in paraffin. For the histological examination, the 6 - $\mu \mathrm{m}$-thick sections were cut at the center of samples and mounted in a micro-slide glass, followed by staining with hematoxylin and eosin and Masson trichrome to observe the cell infiltration, newly formed collagen and new bone formation. Immunostaining for collagen type I antibody was performed to assess the formation of ECM. Antigen retrieval was heat induced in a water bath at $96^{\circ} \mathrm{C}$ for $20 \mathrm{~min}$, with incubation of the slides in citrate buffer $(\mathrm{pH}=6)$. The slides were washed with PBS and endogenous peroxidase was blocked with $0.6 \%$ hydrogen peroxide $\left(\mathrm{H}_{2} \mathrm{O}_{2} ; 31,642\right.$, Sigma, Germany) in methanol (32,213, Sigma, Germany), at room temperature for $30 \mathrm{~min}$. R.T.U. Vectastain ${ }^{\circledR}$ Universal Elite ABC Kit (Vector, VCPK-7200) was used for antibody incubation, according to the instructions of the manufacturer. Briefly, sections were incubated with collagen I antibody (rabbit anti-type I collagen ab292, Abcam, UK) overnight at $4^{\circ} \mathrm{C}$, in a humidified atmosphere. Control sections were incubated with $3 \%$ bovine serum albumin in PBS. After washing with PBS, antibody detection was revealed by using the Peroxidase Substrate Kit DAB (Vector, VCSK4100). Slides were washed in water for $5 \mathrm{~min}$ and then counterstained with Harris' hematoxylin for nuclear contrast. The slides were then mounted using Microscopy Entellan (Merck, NJ, USA) for observation under a light microscope. All images were obtained using a light microscope (Zeiss HAL 100/HBO 100; Axiocam MRc5).

\section{Statistical analysis}

All the experiments were performed with at least three replicates. Results are expressed as mean \pm standard deviation. Differences between the experimental results were analyzed using the one-way analysis of variance, with the Bonferroni's multiple comparison test, defined with a statistical significance as $\mathrm{p}<0.05$.

\section{Results}

Physico-chemical characterization of the empty \& dex-micelles

Micelles presented a spherical morphology, as it can be observed in the AFM image (Figure 1A). Dynamic light scattering showed that the dex-micelles have a mean size of $352 \mathrm{~nm}$ at physiological $\mathrm{pH}$ and a slightly negative zeta potential (Figure 1B). When dispersed in an acidic buffer, the size of the micelles increased significantly up to $595 \mathrm{~nm}$ and zeta potential increased to slightly positive values. The $\mathrm{pH}$ of citrate buffer is similar to the IEP of gelatin, which contributes to the reduced surface charge of the micelles. HPLC analysis showed no free nanoprecipitated drug in the dex-micelles suspension as no distinct peak could be detected for free-form dex.

In vitro cumulative dex release studies were also performed in acidic and neutral conditions (Figure 1C \& D). At $\mathrm{pH} 7.4$, the micelles showed a controlled dex release profile during the tested 7 days, releasing approximately $0.8 \mu \mathrm{g}$ of the corticoid, which corresponds to $40 \%$ of the initial amount of drug. However, when the micelles were dispersed in acidic medium, the release was much faster and all drug was released upon 5 days. 


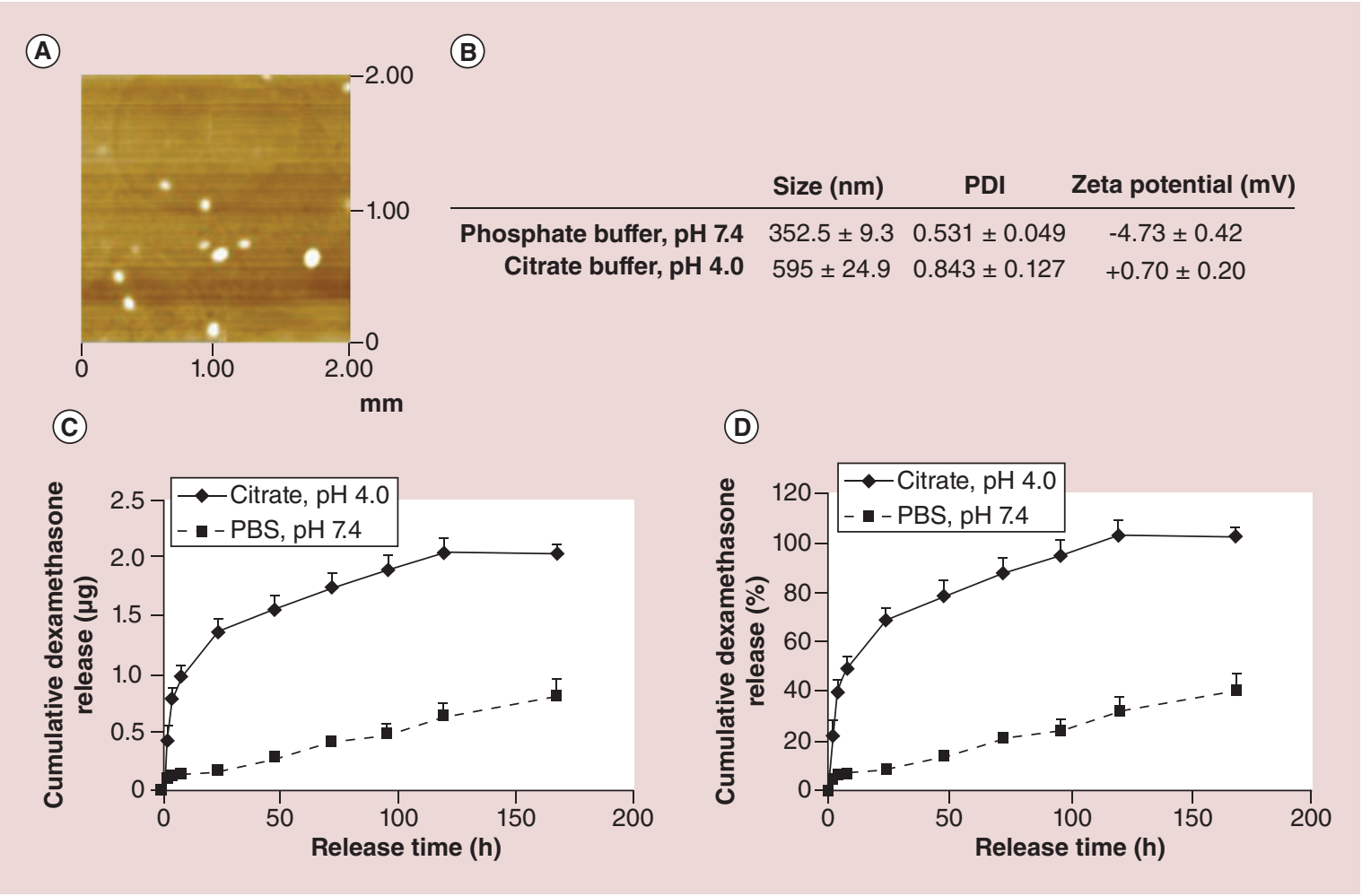

Figure 1. Characterization of the dex-micelles. (A) Morphological characterization by AFM; (B) DLS analysis of the dex-micelles dispersed in neutral and acidic buffers. Parameters such as particle size, PDI and zeta potential were measured. (C \& D) Cumulative in vitro dex release from the drug-loaded micelles in an acidic (- $\bullet$ ) and neutral (--) buffers presented in total mass (C) and\% (D).

AFM: Atomic force microscopy; DLS: Dynamic light scattering; PDI: Particle size distribution index.

\section{Assessment of internalization efficiency of} dex-micelles

The efficiency of fluorescence labeling was assessed and the conjugation of rhodamine to the micelles proved to be effective for the period in which the internalization of the micelles was analyzed $(24 \mathrm{~h})$. The high density of positive cells for fluorescent-labeled micelles was observed for both cell types, in particular for MC3T3E1 cells (Figure 2). Three doses of dex-micelles, for both cell types and for three different incubation periods were tested. For MC3T3-E1 cells, after only $4 \mathrm{~h}$, the levels of internalization almost peaked and remained around $100 \%$ for the remaining incubation periods.

In the case of rBMSCs, the group studies incubated with high doses of fluorescent-labeled dex-micelles showed a statistically significant $(\mathrm{p}<0.05)$ higher number of positive cells for internalization after 4 and $8 \mathrm{~h}$ of incubation, with values around $85 \%$. After $24 \mathrm{~h}$ of incubation with micelles, the cultures containing medium and high doses of dex-micelles showed approximately $90 \%$ of positive cells while the culture with low concentration of dex-micelles showed a statistically significant lower amount of positive cells of only $65 \%$ after $24 \mathrm{~h}$. In addition, Figure 2B shows the fluorescence microscopy images of MC3T3-E1 cells cul- tured with high dose of Rhd-labeled micelles for 8 and $24 \mathrm{~h}$. Micelles are clearly detected in the cytoplasma of the cells, mostly surrounding the cell nucleus. Very few cells were detected presenting absence of internalized micelles, indicating a homogeneous uptake of the nanoparticles by the cell population. Results shown in Figure $2 \mathrm{C}$ demonstrate a significant decrease on micelle internalization when the cultures were incubated with colchicine, an endocytosis-blocking agent. These micelles were shown not to be uptaken by physicochemical permeation through the phospholipic bilayer. Exposure of rBMSCs to colchicine seems to prevent the formation of vesicular structures, suggesting that endocytosis is one of the most important mechanisms of internalization for these nanocarriers.

\section{In vitro biological assessment of dex-micelles}

The incubation of dex-micelles with MC3T3-E1 cells decreased cell proliferation and enhanced in vitro osteogenic differentiation in a dose-dependent manner. In Figure $3 \mathrm{~A}$, it is possible to observe that the addition of empty gelatin micelles did not induce a negative effect on the viability of the cells in both culture mediums in presence or absence of dex. The same pattern was observed for the proliferation studies 
(Figure 3B) up to 5 days of culture and empty micelles showed a similar proliferation profile to osteogenic medium.

At day 7 and 14, some differences could be observed but in general it can be said that the gelatin micelles herein proposed do not harm cell proliferation. However, when cells were loaded with different dosages of dex-micelles (low and high), a significant decrease of cell viability was observed, specifically at days 1, 5 and 7 for all groups and also at day 14 for the cultures with complete osteogenic medium. At day 14, this negative effect was recovered for the cultures in osteogenic medium without dex and the MC3T3-E1 cultures in presence of dex-micelles show similar viability to the controls. Low and high doses of dex-micelles promoted a statistically significant decrease $(p<0.05)$ on cell proliferation at days 5 and 7 when compared with the controls with culture medium only and supplemented with empty micelles. At day 14, this difference is not clear and although there are some individual differences between different group studies, it seems clear that the cells cultured in the presence of dex-micelles recovered their proliferative character.
Supplementary Figure 2A shows the ALP quantification up to 14 days of MC3T3-E1 cells cultured in monolayer with complete osteogenic medium and osteogenic medium without dex. Cells were also supplemented with low and high doses of dex-micelles and it was possible to observe that at day 7 and 14 the ALP activity was significantly enhanced $(p<0.05)$ for the supplemented conditions. Supplementary Figure 2B presents the alizarin red staining for detection of mineralization nodules after 14 days of culture under osteogenic stimulation. Despite the addition of supplements such as ascorbic acid and $\beta$-glycerophosphate, osteogenic medium without dex was not sufficient to promote mineralization and the addition of empty micelles (low and high doses) did not change this outcome. However, when dex-micelles were added to the culture medium without dex, mineralization was clearly enhanced with the formation of several nodules for the group studies where dex-micelles were just added once in the beginning of culture. When fresh dexmicelles were added each time the culture medium was replaced, the mineralization enhancement was

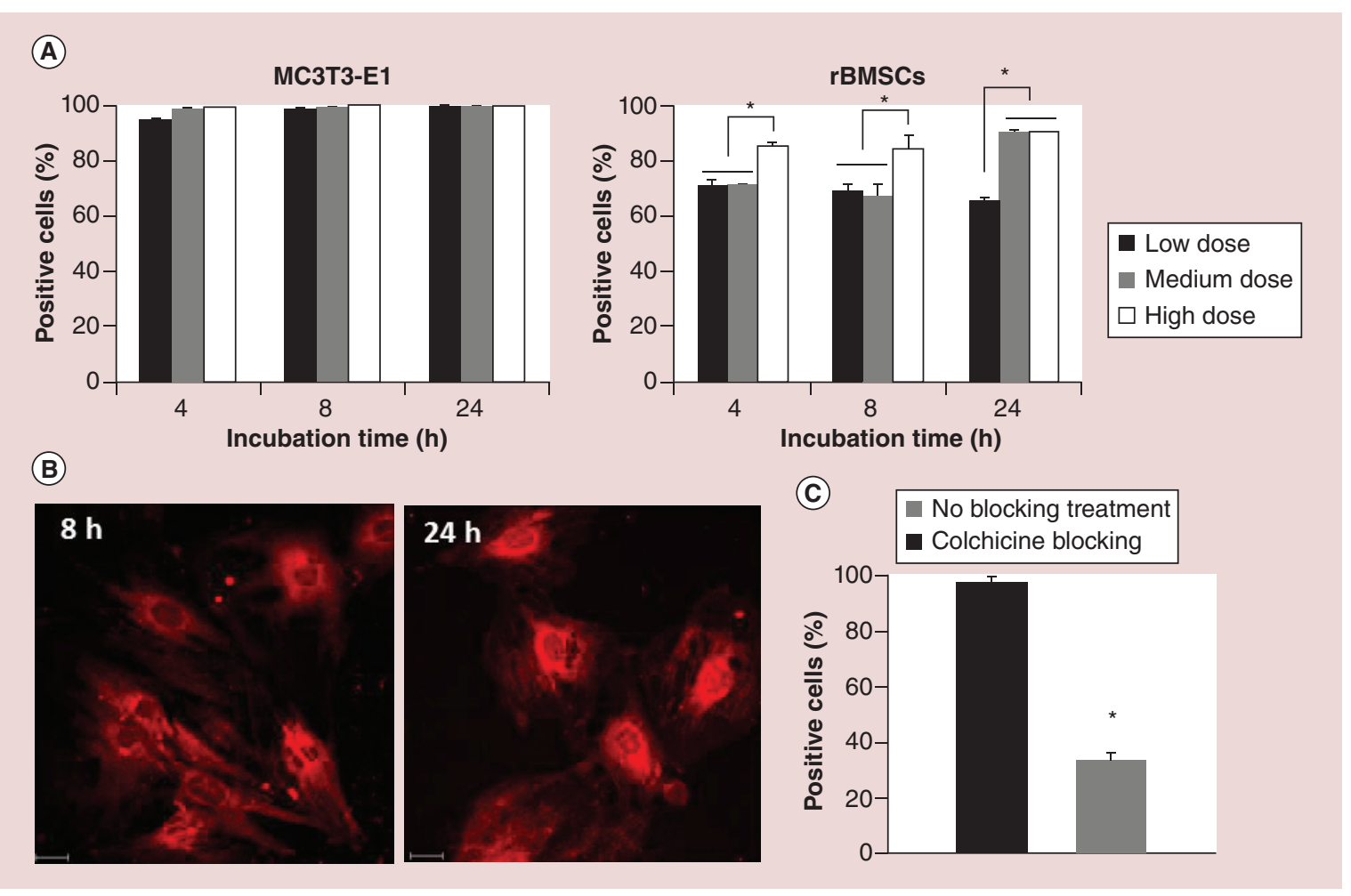

Figure 2. Internalization efficiency of dex-micelles by MС3T3-E1 cells and rat bone marrow-derived mesenchymal stem cells. (A) Flow cytometry analysis of both cell types incubated for 4,8 and $24 \mathrm{~h}$ with three different doses of dex-micelles: low, medium and high doses. ${ }^{*} p<0.05$. (B) Representative images of fluorescence microscopy analysis of MC3T3-E1 cells incubated for 8 and $24 \mathrm{~h}$ with low dose of dex-micelles, correspondent to approximately $100 \%$ of positive cells. Scale bar represents $10 \mu \mathrm{m}$. (C) Flow cytometry analysis of rBMSCs incubated for $24 \mathrm{~h}$ with high dose of dex-micelles in presence or absence of colchicine.

*p $<0.05$.

rBMSC: Rat bone marrow-derived mesenchymal stem cell. 




Figure 3. Evaluation of viability (A) and proliferation (B) of MC3T3-E1 cells cultured 2D in vitro up to 14 days, under osteogenic stimulation. Cells were cultured in complete osteogenic medium in presence (+) or absence (-) of free-form dexamethasone. Two dosages of empty and dex-micelles were assessed - low and high doses. ${ }^{*} \mathrm{p}<0.05$.

less robust and for the high dosage of dex-micelles, it did not even occur. For the MC3T3-E1 cultures in complete osteogenic medium, the addition of dex-micelles significantly decreased the formation of mineralized nodules, both for the group studies where dex-micelles were replaced or not during culture.

Supplementary Figure $2 \mathrm{C}$ reports the ALP quantification of MC3T3-E1 cells seeded onto gelatin hydrogels and cultured up to 14 days in vitro with osteogenic medium without dex. These hydrogels presented a typical porous and interconnected internal structure with pores sized 100-400 $\mu \mathrm{m}$ (see SEM image enclosed in Supplementary Figure 1), which are optimal for bone progenitor cells. Before seeding onto the scaffolds, cells were precultured for $24 \mathrm{~h}$ with three dosages of dexmicelles (low, medium and high) and PBS only as control. A statistically significant $(\mathrm{p}<0.05)$ enhancement of ALP activity of cells precultured with dex-micelles was observed at day 14. Moreover, the increment of ALP activity promoted by the cells precultured with high dose of dex-micelles was significantly stronger $(\mathrm{p}<0.05)$ than the remaining dosages of drug-loaded carriers.

The role of dex dosage can be observed in Supplementary Figure $2 \mathrm{~B}$ in two different scenarios. When the dex-micelles were added to $2 \mathrm{D}$ cultures in complete osteogenic medium (with dex), mineralization was decreased in comparison with the controls; when the dex-micelles were added to $2 \mathrm{D}$ cultures in osteogenic medium without dex, mineralization was strongly enhanced in comparison with the controls; however when fresh dex-micelles were added to the cultures each time the medium was changed, a decrease on the mineralization level was observed for the high-dose condition.

\section{In vivo assessment of bone regeneration in an} ulna defect model

Figure 4A \& B show the soft $x$-ray and micro-CT images of bone regenerated in vivo in the ulna defect, respectively. Figure $4 \mathrm{C}$ presents quantitative data regarding new bone volume formation in vivo.

New bone formation could be observed in the defects implanted with the hydrogels incorporating transplanted cells internalizing dex-micelles. The hydrogels seeded with nontreated rBMSCs did not promote bone regeneration in the defect, as well as the cells precultured with free form dex, as observed in Figure 4A \& B. After 2 and 4 weeks of implantation, it was possible to observe a pattern of more new bone formation when cells were incubated with higher amounts of dex-micelles. The use of a higher amount of cells also enhanced bone formation as observed for the hydrogels seeded with 2 million rBMSCs preincubated with high dose of dex-micelles (Figure 4A \& B). In the control defects, the cut ends of the ulnas were consistently rounded off with a small amount of new bone and bridging of the defects was never observed. In contrast, $\mathrm{x}$-ray and micro-CT images showed progressive infiltration of mineralized tissue into scaffoldtreated defects seeded with cells internalizing dexmicelles, almost completely bridging the defect after 4 weeks.

There was a significant increase in new bone volume with time within treatment groups, as illustrated by Figure $4 C$. The ulna defects treated with gelatin scaffolds seeded with rBMSCs internalizing dex-micelles showed a significant increase $(\mathrm{p}<0.05)$ in bone volume at 2 and 4 weeks of implantation time. Moreover, this tendency was stronger for higher concentrations of micelles incubated with the cells, confirming the results observed by x-ray and micro-CT. The implan- 
tation of gelatin scaffolds with nontreated rBMSCs or rBMSCs precultured with free-form dex did not significantly increase bone volume at either time point. All of the defects bridged by mineralized matrix at 4 weeks had a similar distribution of slightly lower density tissue ingrowth at 2 weeks postimplantation. New bone formation was more intense during the first 2 weeks than the last 2 weeks of implantation time. Figure 5 shows the histological data regarding the in vivo regeneration of the ulna defect and confirmed that the high-density areas seen in the micro-CT scan corresponded to areas of newly formed bone. Two weeks after implantation, histological sections showed new bone formation with osteoblasts and osteo- cytes in all implants containing rBMSCs internalizing dex-micelles. After 4 weeks, more extensive bone formation was observed for the same groups and the implants showed abundant osteocytes with substantial ECM. The gelatin scaffolds seeded with nontreated rBMSCs or precultured with free form dex exhibit fibrous tissue invasion with no evidence of bone formation, demonstrating the critical size of the defect. Small areas of the unfilled defect were still occupied by the remaining implanted gelatin hydrogels (Figure 5). Detailed histological characterization can be observed in Supplementary Figure 4.

Figure 5C \& D (4 weeks implantation time) show a more organized tissue structure than Figure 5A \& B

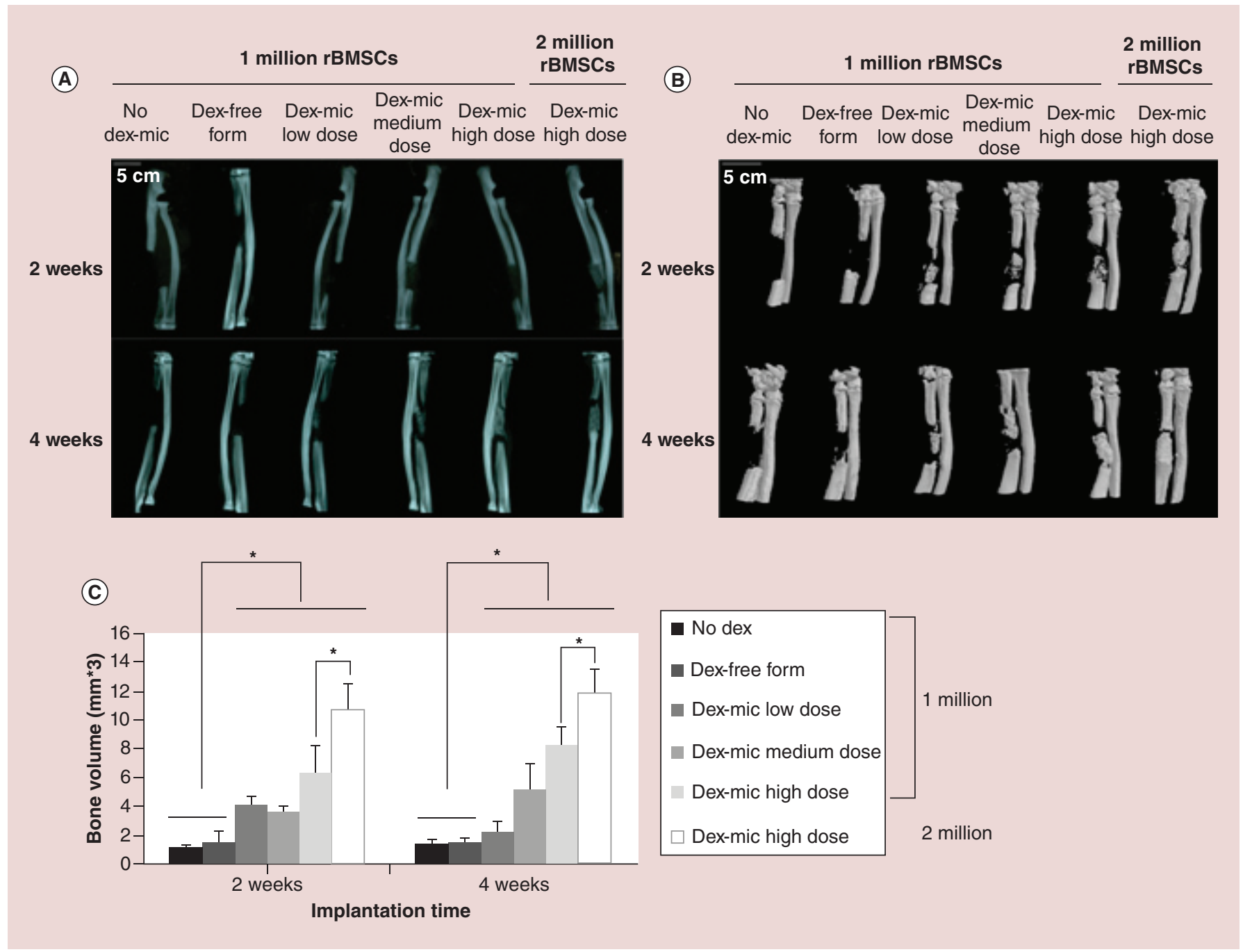

Figure 4. Quantification of new bone tissue formed after 2 and 4 weeks of implantation in a rat ulna critical sized defect of the gelatin constructs seeded with different concentrations of rat bone marrow-derived mesenchymal stem cells precultured for $\mathbf{2 4} \mathrm{h}$ with low $(0.1 \mu \mathrm{g}$ per million of cells), medium ( $0.5 \mu \mathrm{g}$ per million of cells) and high doses ( $1 \mu \mathrm{g}$ per million of cells) of dex-micelles, with free-form dex $(0.1 \mu \mathrm{g}$ per million of cells) and phosphate-buffered saline only as negative controls. The characterization was performed by (A) $\mathrm{x}$-ray; (B) micro-CT and (C) new bone volume measurements, obtained from the micro-CT technique. *p $<0.05$.

rBMSC: Rat bone marrow-derived mesenchymal stem cell. 
(2 weeks implantation time), suggesting the maturation of the new bone tissue being formed and the accumulation of ECM. Supplementary Figure 4 shows the immunohistochemistry staining images of collagen type I (Figure 5A \& C) and negative controls (Figure 5B \& D) for the in vivo regenerated tissue after 2 and 4 weeks of implantation time, respectively. Collagen I detection is more evident for higher doses of dex-micelles.

\section{Discussion}

Previous studies [30,31] demonstrated that the pretreatment of MSCs with dex results in greater bone formation in vivo. However, in vitro osteogenic differentiation requires an additional culture period of weeks [6] and cells may not maintain in vivo the cellular phenotype upon withdrawal of dex, since it acts at multiple stages in the differentiation process [32]. Previous studies regarding the long-term administration of

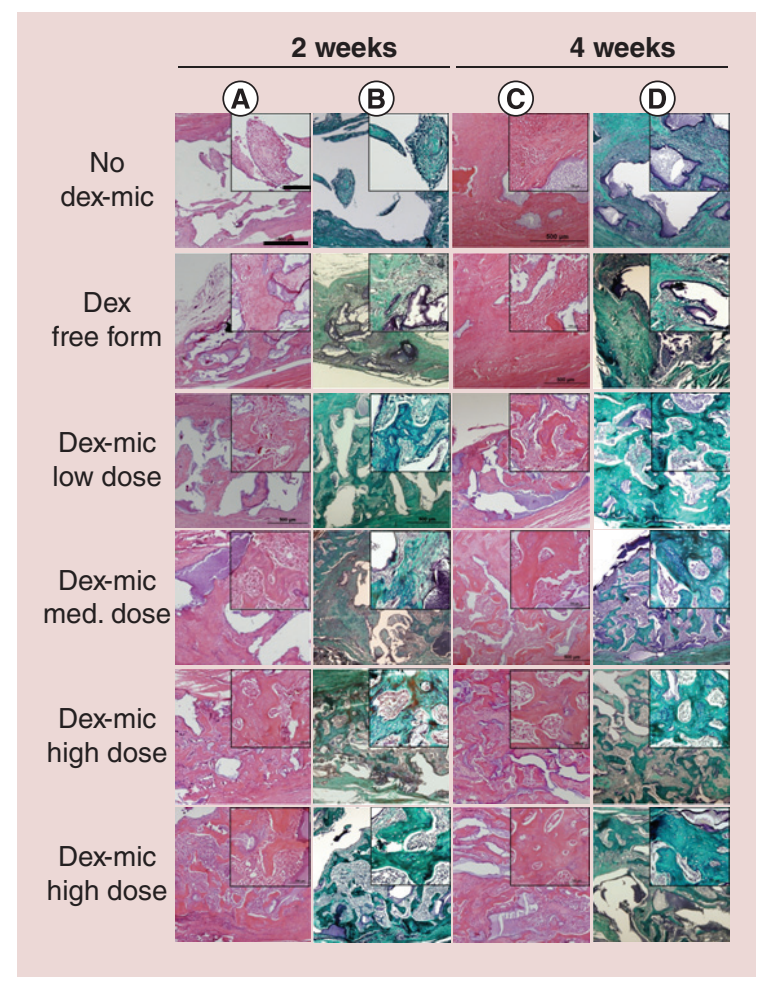

Figure 5. Histological cross-sections of explants from rat ulna defects regenerated with gelatin hydrogels seeded with rat bone marrow-derived mesenchymal stem cells precultured for $\mathbf{2 4} \mathrm{h}$ with low, medium and high doses of dex-micelles, with free-form dex and phosphate-buffered saline only as negative controls. (A) Hematoxylin and eosin and (B) Masson Trichrome, both for 2 weeks after implantation; (C) hematoxylin and eosin and (D) Masson Trichrome for 4 weeks after implantation of the constructs. Embedded images show higher magnifications. Scale bar presents $500 \mu \mathrm{m}$ for the main image and $100 \mu \mathrm{m}$ for the embedded ones. this hormone resulted in the dex-induced bone loss by attenuation of osteoblast proliferation, impaired collagen synthesis, increased osteoblast apoptosis and inhibition of osteogenic growth factors $[8,10]$. Therefore, it is desirable to implant nondifferentiated cells, showing immunomodulatory properties [33,34], supplied with osteoinducer molecules at controlled doses that will enhance their potency for osteogenic differentiation in situ.

The application of a gelatin-based delivery system for a non-water-soluble drug presents several advantages. Gelatin is a biodegradable material and has been extensively used for pharmaceutical and medical purposes [26,35]. The material advantages of gelatin are the easiness of chemical modification and the commercial availability of materials with different physicochemical properties [26]. The micelles herein developed were produced by dialysis through the replacement of the organic medium with water.

At $\mathrm{pH} 4.0$, which is close to the IEP of the gelatin, dex-micelles exhibited an almost neutral surface charge. Negative zeta potential values were recorded for $\mathrm{pH} 7.4$, corresponding also to $\mathrm{pH}$ values above the IEP of gelatin, demonstrating the dependency of the surface charge of gelatin micelles on $\mathrm{pH}$. The narrow particle size distribution index at physiological $\mathrm{pH}$ suggested the narrow size distribution of the dex-micelles at $\mathrm{pH}$ 7.4. However, when dispersed in acidic buffer, PDI increased significantly, suggesting a possible destabilization of the micellar structure. The $\mathrm{pH}$ of 4.0 simulates the acidic environment of the endosomal compartment where the dex-micelles are located after being internalized by the cells. This system presents advantages over previously proposed intracellular delivery systems as it allows the incorporation and release of molecules without covalent conjugation of the drug to the carrier as opposed to dendrimers and drug-polymer conjugate nanocarriers. Moreover, micelles allow the entrapment of higher amounts of drug per nanocarrier, enhancing the efficacy of the release system [36].

Dex-micelles could be bound to fluorescent-probe rhodamine for tracing purposes. The percentage of positive cells internalizing the micelles varied and tended to increase with culturing time up until its peak time. The most significant mechanism of uptake is endocytosis [36] as demonstrated in Figure $2 \mathrm{C}$ and the micelles, at acidic $\mathrm{pH}$ as the acidic and enzymatic environmental $\mathrm{pH}$ of the endosomal compartment, showed a faster release of dex when compared with the release in a neutral buffer. The release of physically encapsulated dex from its carrier is dependent on both the rate of drug diffusion from micellar core and core degradation [37]. In the acidic endosomal compartment, gela- 
tin molecules become protonated which enhances the repulsive forces in the micellar system, widening the mesh sizes of the nanoparticles. Moreover, the acidic environment could lead to a faster degradation of the carrier.

Three different doses of dex-micelles were used during this study. The in vitro $2 \mathrm{D}$ studies were performed only with the two extreme concentrations, low and high doses, while the studies involving the preculture of cells with the dex-micelles for posterior seeding onto gelatin hydrogels for in vitro and in vivo osteogenic differentiation were performed with low, medium and high dosages of dex-micelles. Low dose of dex-micelles corresponded to the same concentration of dex present in osteogenic culture medium $\left(10^{-8} \mathrm{M}\right)$ while medium and high-represented dex concentrations five and tenfold higher, respectively. We hypothesize that undesirable cytotoxicity could be reduced since the intracellular release at acidic $\mathrm{pH}$ also follows a relatively controlled profile, avoiding bolus delivery of dex.

The effect of dex on cell proliferation has been shown to depend on the glucocorticoid concentration and osteoprogenitor maturity [8]. Our results provide evidence of the inhibitory effect of dex over cell proliferation, suggesting that dex acts to direct osteoprogenitor cells from a proliferation state to a matrix maturation stage. At early time points, continuous exposure to dex decreased cell number. These results also showed that dex was in fact being released from the carriers because the conditions where the cultures were supplemented with dex-micelles showed a significant decrease on cell viability and proliferation. This inhibitory effect was reduced at day 14 , which complements the information obtained from the dex in vitro release studies. As dex is fully released from the dex-micelles in an acidic environment after 5 days, cell proliferation was not impaired afterward.

The presence of dex-micelles in culture enhanced ALP activity in a dose-dependent manner (on SI), suggesting stronger osteogenic differentiation of MC3T3-E1 cells. This dose-dependent action of dex has been previously reported $[8,16]$. Despite the addition of osteogenic supplements such as ascorbic acid and $\beta$-glycerophosphate, our in vitro data demonstrated that the delivery of dex by the micelles was required to enhance the levels of osteogenic differentiation. Ascorbic acid is reported to increase the secretion of collagen I and consequently leading to the phosphorylation of ERK1/2 in the MAPK signaling pathway and subsequent translocalization of ERK1/2 to the nucleus, where it binds to Runx2 and induces gene expression of osteogenic proteins. On the other hand, $\beta$-glycerophosphate acts as the source of phos- phate for hydroxyapatite and on the phoshorylation of ERK1/2 [38]. Despite the significant contribution of ascorbic acid and $\beta$-glycerophosphate for in vitro osteogenic differentiation, results from this study demonstrate that their supplementation in the culture medium is not sufficient to trigger a transition from a precursor to a fully differentiated osteoblastic phenotype.

Gelatin hydrogels have been shown to act as osteoconductive biomaterial in vivo [26] and to provide an affective template for new bone growth and defect healing in this defect model [39]. MC3T3-E1 osteoblast-like cells precultured for $24 \mathrm{~h}$ with three dosages of dex-micelles and seeded onto gelatin hydrogels presented indication of stronger osteogenic induction by the enhancement of ALP activity in vitro. Therefore the ability of dex-micelles to enhance osteogenic differentiation was kept in a 3D setting with only a 24-h preculture supplement.

The orthotopic location in vivo is expected to provide more accurate outcomes concerning the influence or local effects of implanted cells or cell scaffold constructs where they were initially designed to be functional [40]. The ulna defect is appropriate for in vivo tests as it does not require further internal or external fixation, which might influence the outcome of the experiment. This model, including the specific dimensions of the defect $(6 \mathrm{~mm})$ was previously validated, since it was shown to enable a minimal growth of bone volume in the defect over time [27]. For the in vivo studies described in the present study, hydrogel samples were seeded with 1 million of rBMSCs, precultured with low, medium and high doses of dexmicelles. As controls, cell precultures were supplemented with PBS only and free-form dex $\left(10^{-8} \mathrm{M}\right)$. An additional group study was added with a seeding of 2 million cells (precultured with high doses of dex-micelles) per scaffold. The selected implantation times, 2 and 4 weeks, were chosen based on previous studies using the same defect model and gelatin hydrogels $[27,28]$. Since an early action of dex was expected, due to the natural role of the drug, in vitro release profile and general instability of the micelles in an acidic environment, the implantation times were thought to be adequate.

It was also shown that an in vitro osteogenic induction of BMSCs prior to the implantation facilitates bone formation in the implanted constructs [34]. However, it has been stated that the withdrawal of dex from the cultures when implanted in vivo resulted in an inferior osteogenic differentiation. The previous incubation of the cells with the loaded micelles permits to program their differentiation to the osteoblastic lineage even if they are placed in non- 
osteogenic conditions. Therefore, the preculture of osteoprogenitor cells with dex-micelles for $24 \mathrm{~h}$ and further seeding onto a gelatin construct with implantation in vivo of the construct at day 0 is a feasible strategy to promote new bone formation in a critical size defect and to overcome a long preimplantation in vitro osteogenic induction. The rBMSCs would still present their stemness character while internalizing an osteoinducer molecule, reducing the preculture time and avoiding dex withdrawal after preculture while stimulating rBMSCs toward the osteogenic phenotype. Moreover, the use of high concentrarions of dex in free form as the ones selected for the highest dose of dex-micelles proved to be toxic for the rBMSCs, leading to significant decrease of cell viability after few hours of incubation with the glucocorticoid. This highlights the potential to use the gelatin-based micelles in order to control the delivery kinetics of a potent osteoinducer but toxic molecule such as dex.

The production of calcium phosphate mineral phase detected by $\mathrm{x}$-ray and micro-CT suggests the generation of a mature osteoblast population, which allows the production of osteoid and ECM [7]. The x-rays, micro-CT and new bone volume quantifications showed a progressive increase of mineralized tissue with increasing dosages of dex-micelles. This suggests that more dex-micelles were internalized by the cells and that they are releasing the drug and promoting the formation of new mineralized tissue. The constructs seeded with nontreated rBMSCs did not regenerate the defect, confirming its critical size. Preculture with freeform dex also did not promote new bone formation. For this group study, rBMSCs were in contact with the glucocorticoid only for $24 \mathrm{~h}$ and then the drug was withdrawn, while the dex-micelles were expected to release dex inside the cells up to almost 1 week post-in vivo implantation.

In vivo bone regeneration induced by BMSCs depends on the presence of a sufficient number of early osteoprogenitors on the implant that can proliferate and further differentiate into osteoblasts [15]. For this reason, the cell number was also evaluated and two cell densities were assessed: 1 and 2 million of rBMSCs per scaffold. New bone volume was significantly higher for the constructs seeded with 2 million rBM$\mathrm{SCs}$ and a bigger cell population was more effective for the formation of new bone tissue.

The therapeutic action of donor MSCs may not be solely limited to their direct conversion into osteoblasts as they also possess other functionalities such as antiapoptotic effects, immunoregulatory functions and stimulation of host cell migration [41]. Therefore, the implanted MSCs can act as signaling centers for the host response to the injury and play a 'kick-off' role in attracting other cells from the host by the secretion of paracrine factors and stimulate them to differentiate toward the osteogenic lineage. In the literature it is not reported the role of dex as a chemotactic agent for host MSCs, which allows us to speculate that the corticoid is mainly acting on the osteogenic differentiation of the implanted cells, which then, through intercellular mechanisms, induce an enhanced host MSCs infiltration within the defect. The clear observation of the drug concentration pattern on new bone formation and the increased regenerated tissue in implants with higher cell amount suggest that the implanted cells are responsible for the differences between the different groups. These results show that a longer supply of dex in a regimented manner, as the corticoid can be released from the micelles inside the cells during the in vivo regeneration, promotes an enhancement of new bone formation. However, it should be stated that dex can raise the risk of infection and therefore careful monitoring should be performed in order to avoid infection risks during tissue repair procedures.

The results obtained in this study were at least comparable to the bone regeneration observed for the same model using the delivery of bone morphogenetic protein-2 [27], gold standard for the promotion of new bone formation. This outcome demonstrates that the design of appropriate nanoparticle and cell engineering strategies can improve some of the limitations observed with current delivery systems, which include excessive dosages, high cost and inefficient drug action. Cell engineering is a powerful tool to manipulate cell fate and differentiation and the proposed drug delivery mechanism is a promising approach for bone regeneration.

\section{Conclusion}

In summary, the developed dex-micelles were able to be internalized by MC3T3-E1 osteoblast-like cells and rBMSCs and to release dex in a $\mathrm{pH}$-dependent manner. The drug-loaded micelles promoted the $2 \mathrm{D}$ in vitro osteogenic differentiation of MC3T3-E1 and their preculture with dex-micelles for $24 \mathrm{~h}$ prior seeding onto gelatin hydrogels also resulted in enhanced osteogenic differentiation. Moreover, when in an in vivo setting, the rBMSCs precultured with dexmicelles for $24 \mathrm{~h}$ and seeded onto gelatin hydrogels promoted a superior in vivo bone formation due to the intracellular release of dex, which enhanced rBMSCs osteogenic differentiation in a dose-dependent fashion.

This study shows that the combination of cell engineering, nanotechnology and tissue engineering in one 
strategy is a powerful tool to manipulate cell fate and differentiation and the proposed drug delivery mechanism is a promising approach for bone regeneration. Moreover, this system has the potential for application in cell engineering approaches for other tissues, in which the released bioactive agent might modulate the cellular behavior through the action on cytoplasmatic receptors.

\section{Future perspective}

The development of new technologies and the ability to build up tissue engineering and regenerative medicine strategies with higher levels of complexity and characterization has been increasingly observed for the past decade. The use of targeted nanocarriers for drug delivery to preprogram stem cells in order to modulate their fate and performance represents a promising approach for maximizing the potential of these regenerative therapies. Critical considerations for the development of improved carriers include safety, biocompatibility, stability and the ability to transfer therapeutic molecules to the target cells at high efficiency.

It is crucial to understand the barriers that need to be overcome during the transport process in order to develop highly efficient delivery systems. The biological fate of nanoparticles in the body is one of the main concerns in this field of research and increasing efforts have been invested to comprehend the mechanisms of internalization and excretion of these carriers from the cells, as well as their distribution in the body and affinity for specific tissues. The toxicity assessment is still at an early stage of development and inconsistent data are reported in the literature due to the lack of standardization of protocols for the assessment of toxicity of nanomaterials. Moreover, in order to support smooth transition into clinical practice, the ease of large-scale manufacture, administration and cost-effectiveness must also be considered.

\section{Author contributions}

VE Santo and Y Tabata designed research; VE Santo, K Sato and J Ratanavaraporn performed research; VE Santo, J Ratanavaraporn, ME Gomes, JF Mano, RL Reis and Y Tabata were involved in the interpretation of the data and revised the manuscript; VE Santo wrote the manuscript.

\section{Acknowledgements}

The authors gratefully acknowledge ARC Duarte, Mariana Cerqueira, Nélson Oliveira, Luís Martins and António Salgado for technical assistance.

\section{Financial \& competing interests disclosure}

The authors thank Fundação para a Ciência e Tecnologia and Japan Society for the Promotion of Science (JSPS) for VE Santo's PhD grant (SFRH/BD/39486/2007) and J Ratana-

\section{Executive summary}

Production \& development of dexamethasone-loaded gelatin micelles

- Gelatin micelles presented a pH-dependent release profile of dexamethasone (dex).

- The micelles showed the ability to be internalized by different cell types in few hours, with levels of efficiency between 65 and $100 \%$.

- When blocking the endocytic route, the levels of micelle internalization were significantly reduced, thus suggesting endocytosis as one the main mechanisms by which the particles were internalized.

In vitro biological assessment of dexamethasone-loaded gelatin micelles

- Exposure of cells to dex-loaded nanocarriers induced an inhibition on cell proliferation, thus demonstrating that the drug was indeed being released from the micelles. This effect was time and dose-dependent as at later time points, cells were able to recover their proliferative character once dex was fully released from the nanoparticles.

- Incubation of osteoprogenitor cells with the dex-loaded micelles enhanced alkaline phosphatase production and deposition of mineralized matrix.

In vivo biological assessment of dexamethasone-loaded gelatin micelles

- Preculture of rat bone marrow stem cells with dex-loaded micelles for $24 \mathrm{~h}$ and further seeding of those cells onto gelatin hydrogels and consequent implantation in vivo in rat ulna critical size defects resulted in an efficient deposition of mineralized tissue up to 4 weeks of implantation.

- The extent of regenerated bone was dependent on the dosage of micelles that were preincubated with the stem cells, as well as the concentration of stem cells seeded onto the gelatin scaffolds.

- Histological analysis revealed that the newly formed tissue already presented high levels of bone extracellular matrix organization, unlike the controls with empty micelles or free-form dex, in which only fibrotic tissue could be found.

- This strategy is a cost-effective and efficient cell engineering approach to induce bone regeneration as by tailoring the properties and dosages of the delivery systems, high levels of tissue regeneration were quickly achieved. 
varaporn's post-doc fellowship, respectively. The authors have no other relevant affiliations or financial involvement with any organization or entity with a financial interest in or financial conflict with the subject matter or materials discussed in the manuscript apart from those disclosed.

No writing assistance was utilized in the production of this manuscript.

\section{References}

Papers of special note have been highlighted as: • of interest

1 Petite H, Viateau V, Bensaid W et al. Tissue-engineered bone regeneration. Nat. Biotechnol. 18(9), 959-963 (2000).

2 Caplan AI, Bruder SP. Mesenchymal stem cells: building blocks for molecular medicine in the 21st century. Trends Mol. Med. 7(6), 259-264 (2001).

3 Dupont KM, Sharma K, Stevens HY, Boerckel JD, Garcia AJ, Guldberg RE. Human stem cell delivery for treatment of large segmental bone defects. Proc. Natl Acad. Sci. USA 107(8), 3305-3310 (2010).

4 Horwitz EM, Prockop DJ, Fitzpatrick LA et al. Transplantability and therapeutic effects of bone marrowderived mesenchymal cells in children with osteogenesis imperfecta. Nat. Med. 5(3), 309-313 (1999).

5 Quarto R, Mastrogiacomo M, Cancedda R et al. Repair of large bone defects with the use of autologous bone marrow stromal cells. N. Engl. J. Med. 344(5), 385-386 (2001).

6 Kang SW, La WG, Kang JM, Park JH, Kim BS. Bone morphogenetic protein-2 enhances bone regeneration mediated by transplantation of osteogenically undifferentiated bone marrow-derived mesenchymal stem cells. Biotechnol. Lett. 30(7), 1163-1168 (2008).

7 Dalby MJ, Gadegaard N, Tare R et al. The control of human mesenchymal cell differentiation using nanoscale symmetry and disorder. Nat. Mater. 6(12), 997-1003 (2007).

8 Porter RM, Huckle WR, Goldstein AS. Effect of dexamethasone withdrawal on osteoblastic differentiation of bone marrow stromal cells. J. Cell. Biochem. 90(1), 13-22 (2003).

- This study indicates that dexamethasone acts both at early and late stages to direct proliferative osteoprogenitor cells toward terminal maturation.

9 Mikami Y, Omoteyama K, Kato S, Takagi M. Inductive effects of dexamethasone on the mineralization and the osteoblastic gene expressions in mature osteoblast-like ROS17/2.8 cells. Biochem. Biophys. Res. Commun. 362(2), 368-373 (2007).

10 Phillips JE, Gersbach CA, Wojtowicz AM, Garcia AJ. Glucocorticoid-induced osteogenesis is negatively regulated by Runx2/Cbfa1 serine phosphorylation. J. Cell Sci. 119(Pt 3), 581-591 (2006).

11 Advani S, Lafrancis D, Bogdanovic E, Taxel P, Raisz LG, Kream BE. Dexamethasone suppresses in vivo levels of bone collagen synthesis in neonatal mice. Bone 20(1), 41-46 (1997).

$12 \mathrm{Kim} \mathrm{H}$, Suh H, Jo SA et al. In vivo bone formation by human marrow stromal cells in biodegradable scaffolds that release
Ethical conduct of research

The authors state that they have obtained appropriate institutional review board approval or have followed the principles outlined in the Declaration of Helsinki for all human or animal experimental investigations. In addition, for investigations involving human subjects, informed consent has been obtained from the participants involved.

dexamethasone and ascorbate-2-phosphate. Biochem. Biophys. Res. Commun. 332(4), 1053-1060 (2005).

13 Hamidouche Z, Hay E, Vaudin P et al. FHL2 mediates dexamethasone-induced mesenchymal cell differentiation into osteoblasts by activating Wnt/beta-catenin signalingdependent Runx2 expression. FASEB J. 22(11), 3813-3822 (2008).

14 Hong D, Chen HX, Xue Y et al. Osteoblastogenic effects of dexamethasone through upregulation of TAZ expression in rat mesenchymal stem cells. J. Steroid Biochem. Mol. Biol. 116(1-2), 86-92 (2009).

15 Mendes SC, Tibbe JM, Veenhof M et al. Relation between in vitro and in vivo osteogenic potential of cultured human bone marrow stromal cells. J. Mater. Sci. Mater. Med. 15(10), 1123-1128 (2004).

16 Oliveira JM, Kotobuki N, Tadokoro M et al. Ex vivo culturing of stromal cells with dexamethasone-loaded carboxymethylchitosan/poly(amidoamine) dendrimer nanoparticles promotes ectopic bone formation. Bone 46(5), 1424-1435 (2010).

17 Oliveira JM, Sousa RA, Malafaya PB et al. In vivo study of dendronlike nanoparticles for stem cells "tune-up": from nano to tissues. Nanomedicine 7(6), 914-924 (2011).

18 Santo VE, Rodrigues MT, Gomes ME. Contributions and future perspectives on the use of magnetic nanoparticles as diagnostic and therapeutic tools in the field of regenerative medicine. Expert Rev. Mol. Diagn. 13(6), 553-566 (2013).

19 Richardson TP, Peters MC, Ennett AB, Mooney DJ. Polymeric system for dual growth factor delivery. Nat. Biotechnol. 19(11), 1029-1034 (2001).

20 Santo VE, Gomes ME, Mano JF, Reis RL. From nano- to macro-scale: nanotechnology approaches for spatially controlled delivery of bioactive factors for bone and cartilage engineering. Nanomedicine (Lond.) 7(7), 1045-1066 (2012).

21 Chen H, Khemtong C, Yang X, Chang X, Gao J. Nanonization strategies for poorly water-soluble drugs. Drug Discov. Today 16(7-8), 354-360 (2011).

22 Sahay G, Batrakova EV, Kabanov AV. Different internalization pathways of polymeric micelles and unimers and their effects on vesicular transport. Bioconjug. Chem. 19(10), 2023-2029 (2008).

23 Salgado AJ, Oliveira JM, Pirraco RP et al. Carboxymethylchitosan/poly(amidoamine) dendrimer nanoparticles in central nervous systems-regenerative medicine: effects on neuron/glial cell viability and internalization efficiency. Macromol. Biosci. 10(10), 1130-1140 (2010).

24 Wang Q, Wang J, Lu Q, Detamore MS, Berkland C. Injectable PLGA based colloidal gels for zero-order 
dexamethasone release in cranial defects. Biomaterials 31(18), 4980-4986 (2010).

Oliveira JM, Sousa RA, Kotobuki N et al. The osteogenic differentiation of rat bone marrow stromal cells cultured with dexamethasone-loaded carboxymethylchitosan/ poly(amidoamine) dendrimer nanoparticles. Biomaterials 30(5), 804-813 (2009).

Tanigo T, Takaoka R, Tabata Y. Sustained release of waterinsoluble simvastatin from biodegradable hydrogel augments bone regeneration. J. Control. Release 143(2), 201-206 (2010).

- Report highlighting the use of micelles for the delivery of drugs with clear stimulation of in vivo bone regeneration.

27 Ratanavaraporn J, Furuya H, Kohara H, Tabata Y. Synergistic effects of the dual release of stromal cell-derived factor- 1 and bone morphogenetic protein-2 from hydrogels on bone regeneration. Biomaterials 32(11), 2797-2811 (2011).

28 Yamamoto M, Takahashi Y, Tabata Y. Controlled release by biodegradable hydrogels enhances the ectopic bone formation of bone morphogenetic protein. Biomaterials 24(24), 4375-4383 (2003).

29 Virk MS, Conduah A, Park SH et al. Influence of short-term adenoviral vector and prolonged lentiviral vector mediated bone morphogenetic protein-2 expression on the quality of bone repair in a rat femoral defect model. Bone 42(5), 921-931 (2008).

30 Song IH, Caplan AI, Dennis JE. In vitro dexamethasone pretreatment enhances bone formation of human mesenchymal stem cells in vivo. J. Orthop. Res. 27(7), 916-921 (2009).

31 Yoon E, Dhar S, Chun DE, Gharibjanian NA, Evans GR. In vivo osteogenic potential of human adipose-derived stem cells/poly lactide-co-glycolic acid constructs for bone regeneration in a rat critical-sized calvarial defect model. Tissue Eng. 13(3), 619-627 (2007).

32 Nuttelman CR, Tripodi MC, Anseth KS. Dexamethasonefunctionalized gels induce osteogenic differentiation of encapsulated hMSCs. J. Biomed. Mater. Res. A 76(1), 183-195 (2006).

33 Berner A, Reichert JC, Muller MB et al. Treatment of long bone defects and non-unions: from research to clinical practice. Cell Tissue Res. 347(3), 501-519 (2012).

34 Cancedda R, Giannoni P, Mastrogiacomo M. A tissue engineering approach to bone repair in large animal models and in clinical practice. Biomaterials 28(29), 4240-4250 (2007).

35 Ofokansi K, Winter G, Fricker G, Coester C. Matrixloaded biodegradable gelatin nanoparticles as new approach to improve drug loading and delivery. Eur. J. Pharm. Biopharm. 76(1), 1-9 (2010).

36 Blanco E, Kessinger CW, Sumer BD, Gao J. Multifunctional micellar nanomedicine for cancer therapy. Exp. Biol. Med. (Maywood) 234(2), 123-131 (2009).

37 Xiong XB, Mahmud A, Uludag H, Lavasanifar A. Multifunctional polymeric micelles for enhanced intracellular delivery of doxorubicin to metastatic cancer cells. Pharm. Res. 25(11), 2555-2566 (2008).

Langenbach F, Handschel J. Effects of dexamethasone, ascorbic acid and beta-glycerophosphate on the osteogenic differentiation of stem cells in vitro. Stem Cell Res. Ther. 4(5), 117 (2013)

39 Tadokoro M, Matsushima A, Kotobuki N et al. Bone morphogenetic protein-2 in biodegradable gelatin and beta-tricalcium phosphate sponges enhances the in vivo bone-forming capability of bone marrow mesenchymal stem cells. J. Tissue Eng. Regen. Med. 6(4), 253-260 (2012).

40 Rodrigues MT, Gomes ME, Viegas CA et al. Tissueengineered constructs based on SPCL scaffolds cultured with goat marrow cells: functionality in femoral defects. J. Tissue Eng. Regen. Med. 5(1), 41-49 (2011).

41 Jones E, Yang X. Mesenchymal stem cells and bone regeneration: current status. Injury 42(6), 562-568 (2011). 